\title{
Metabolic Process Engineering for Biochemicals and Biofuels Production
}

\section{Shang-Tian Yang ${ }^{1 *}$ and Xiaoguang Liu $^{2 *}$}

${ }^{1}$ Department of Chemical and Biomolecular Engineering, The Ohio State University, USA ${ }^{2}$ Department of Chemical and Biological Engineering, The University of Alabama, USA

Keywords: Metabolic process engineering; Biofuel; Biochemical; Microbial fermentation

\section{Introduction}

The trends towards using green chemical and energy are increasing due to the growing demand for non-fossil bio-products, the environmental concern for using fossil fuel, and the continuously increasing cost of crude oils. The total annual markets of biofuels and biochemicals are estimated to exceed \$1 trillion[1]. Microbial fermentation has been widely used to produce organic biochemicals and biofuels, including citric acid, lactic acid, butyric acid, propionic acid, amino acids, ethanol, propanol, butanol, etc. The rapidly growing biotechnology market requires an efficient bioprocess platform, including both the production cell and the process, for biochemicals and biofuels production. Metabolic engineering (ME) is often used to develop high-producing cells needed for the process. However, ME requires genetically modifying the cell, which can be difficult to do or to achieve the expected outcome, especially for less studied microorganisms. Metabolic process engineering (MPE) is a novel and advanced technology that alters or manipulates metabolic pathway to produce the interested metabolites by rationally controlling or manipulating bio-production process parameters. The goal of MPE is to achieve a high-productivity, high-quality, robust and scalable process through dynamic monitoring and investigating the interactions between cellular metabolism and process parameters. Different from the well-known traditional fermentation process development, MPE targets to engineer the bio-production process by controlling the cell physiology and metabolic responses to changes in fermentation process parameters and incorporating the interplay between cell and process into the rational process design. In this article, we focus on the application of MPE to improve biochemicals and biofuels production via precise bioreactor controllers, in situ sensors, and omics technologies.

Fermentation can be disturbed by slight changes in some process parameters, which leads to variable product quality. The major process parameters in fermentation include bioreactor operation parameters and metabolic process parameters. The bioreactor operation parameters (e.g., agitation rate, temperature, $\mathrm{pH}$ and $\mathrm{DO}$ ) can be controlled by precise bioreactor controllers and in situ probes, which have been well evaluated and developed in process development in the biotechnology industry. For example, a fuzzy-PI controller has been developed to maintain a precise temperature by controlling temperature variation within a narrow range in large scale ethanol production [2]. The mathematic modelling has been successfully developed to assess the dynamic behaviour of bio-butanol fermentation consisting of various interconnected units such as fermenter, cell retention system, and vacuum vessel[3].However, it is hard to directly regulate or manipulate metabolic process parameters (e.g., basal medium, substrate, feed rate and feed formulation) in fed-batch fermentation due to complicated and dynamically variable metabolic activities of microorganism.

Fed-batch fermentation has been widely used in biochemical, biofuel and food industries. The optimal nutrient feeding strategy can main certain cell growth to support bio-production, avoid nutrient depletion to achieve high volumetric productivity, and minimize the accumulation of by-products. One challenge to optimize feeding strategy (e.g., feeding rate and feed formulation) is how to collect and analyse dynamic metabolic parameters, including biomass, extracellular metabolites (substrate, product, by-product, and other metabolite) and intracellular metabolites. Although HPLC, GC, MS and other analytical technologies have been applied to analyse substrates and products offline, the profiling of intracellular and extracellular metabolites (e.g. the interested intermediates and end metabolite in key metabolic pathway), varies in the course of time. Robust, fast response, precise and in situ probes can partially solve this issue by providing online sample analysis. Some sensors, including biomass probe, dissolved oxygen probe, extracellular oxidoreduction potential probe, and gas monitors, are used in fermentation to monitor cell growth, assess aerobic metabolism, estimate $\mathrm{NAD}^{+} / \mathrm{NADH}^{+}$ratio, and measure gases (e.g., $\mathrm{CO}_{2}, \mathrm{CH}_{4}$ and $\mathrm{H}_{2}$ ), respectively[4].In addition, biochemical analysers connected to auto-samplers are used for online monitoring of multiple metabolites. Different from discrete sensors, the MS based chemical multisensory systems, named as electronic noses and electronic tongues [5], have been used in both qualitative recognition of multi-component media and quantitative analysis of component concentrations in wine production. With dynamic metabolite data collected using in situ probes and auto-samplers, mathematic modelling can be applied to achieve precise nutrients feed and harvest time in fedbatch fermentation. For example, the maximum or dynamic substrate feed rate in aerobic fermentation has been determined by developing a feeding model that correlates substrate mass transfer and substrate uptake to volumetric oxygen transfer rate [6].

High-productivity fermentation processes have been developed using traditional bioreactor controller and metabolic process development tools. However, the rational design of a metabolically engineered fermentation process to achieve high-productivity, highquality, and high-robustness is far behind in the biotechnology industry. This is caused by the lack of a fundamental understanding of the interaction between cellular activities and fermentation

*Corresponding authors: Shang-Tian Yang, Professor, Department of Chemical and Biomolecular Engineering, The Ohio State University, 140 West 19th Avenue Columbus, OH 43210 USA, Tel: (614) 292-6611; Fax: (614) 292-3769; E-mail: Yang.15@osu.edu

Xiaoguang Liu, Assistant Professor, Department of Chemical and Biological Engineering, The University of Alabama, 245 7th Avenue, Tuscaloosa, AL 35401 USA, Tel: (205) 348-0868; Fax: (205) 348-7558; E-mail: mliu@eng.ua.edu

Received January 18, 2014; Accepted January 20, 2014; Published January 24 2014

Citation: Yang ST, Liu X (2014) Metabolic Process Engineering for Biochemicals and Biofuels Production. J Microb Biochem Technol 6: e116. doi:10.4172/19485948.1000e116

Copyright: (C) 2014 Yang ST, et al. This is an open-access article distributed under the terms of the Creative Commons Attribution License, which permits unrestricted use, distribution, and reproduction in any medium, provided the original author and source are credited 
environment. The recent advances in omics technologies enable the fermentation process profiling, and thereby provides an in-depth understanding of genome background, global protein profiling, and metabolite map of bio-production. Omics studies usually refer to genomics, transcriptomics, proteomics, metabolomics and others. 1) Genomics is the comprehensive and complete analysis of genome using new-generation DNA sequencers such as Illumina Hi Seq 2000 or Life Tech SOLiD; 2) Transcriptomics is a functional genomics analysis by qualifying and quantitating messenger RNA using next-generation sequencing technologies; 3 ) Proteomics is to quantitate the expression of intracellular proteins under defined culture conditions using SELDITOF-MS, UPLC-MS/MS and MALDI-TOF-MS; and4) Metabolomics is to identify and quantify a large number of cellular metabolites using LC-MS.

Omics have been recently used in the biotechnology industry to develop fundamental understanding of the phenotype in biobutanol and biochemical production. For example, transcriptomics has been used to analyse the response of Clostridium acetobutylicum ATCC 824 to butanol stress, which generated a new medium formulation to maintain high cell growth and butanol production [7]. Another genome-wide transcriptional analysis with the next-generation sequencing technology has been performed to investigate the effect of butyrate supplement on butanol metabolic switch in C. beijerinckii NCIMB 8052 [8]. With the access and integration with genomics database and transcriptomics knowledge, it is feasible to identify metabolites, establish metabolic reactions, and reconstruct metabolic networks via metabolomics. The core metabolites responsible for carbon, energy and redox balance, amino acids, end product inhibition and cell growth under defined culture conditions or production processes can also be distinguished.

Metabolomics is a powerful approach in MPE because it is capable of finding the regulatory mechanism of metabolic flux balance or regulation. Metabolic flux reveals the overall outcome of various cellular components, such as genes, transcripts, proteins, and metabolites, and interplayed factors, such as gene regulation, proteinprotein interaction, and metabolic network. Therefore, the metabolic flux analysis facilitated with metabolomics approach is the key to MPE. The increasing metabolic coverage and analytical resolution in metabolomics provides the direct evaluation of pathway intermediates. Multiple software tools (e.g., Open Flux and Fiat-Flux) are available and allow for user-friendly metabolic flux calculation by integrating experimental metabolomics data $[9,10]$. The metabolic network can be constructed using statistical analysis such as unsupervised learning, correlation network analysis, pattern recognition, principle component analysis, or dynamics control theory [11].

With the rapid advancement of systems biology, a large amount of metabolomics data has been accumulated and some well-known public metabolic pathway databases have been created, such as, MetaCyc, Kyoto Encyclopedia of Genes and Genomes (KEGG), Pathway Interaction Database (PID), Reactome and WikiPathway [12]. Some de novo models have been developed to facilitate data interpretation, but they rely on the literature mining and manual processing, so it is still challenging to extract key information from the big data[13]. To solve this issue, Buchel, et al. have established Path2Models database by including kinetic, logical, rule-based, multi-agent, constraint-based and statistical models [14]. The advantage of Path2Models database is that it can automatically generate mathematical model from pathway data sources, such as KEGG, Bio Carta, Meta Cyc and SABIO-RK. Various types of models have been developed based on the Path2Models and shared through Bio Modes Database and the Cell ML repository [11].In addition to these databases and models, computational systems biology modelling will be a good strategy to perform functional analysis and infer cellular network, which integrates various statistical frameworks and mathematical formulas [13].

With the continuing market growth for microorganism-based biochemicals and biofuels, it is of great interest for the biotechnology industry to rationally design effective bioprocesses. Rational design requires the accurate prediction of cell responses to changes in fermentation conditions. The rational process design empowered by omics technologies, especially transcriptomics and metabolomics, allows for investigating gene expression, developing metabolite profiling, distinguishing metabolic regulators, and identifying critical process parameters. Therefore, the integration of rational design with omics technologies in MPE can contribute to the development of metabolically engineered processes for industrial production of biochemicals and biofuels with high productivity and high product quality.

In summary, MPE is a powerful technology that integrates the well-developed process control techniques, such as precise bioreactor controllers and in situ sensors, and advanced omics technologies. MPE enables the rational design of a bio-production process, and thus can lead to a highly efficient fermentation process for biochemicals and biofuels production. MPE not only can contribute to the enhanced production of metabolites in fermentation but also can provide an indepth understanding of interplays between cells and the fermentation process. Current metabolic engineering approaches require genetically modifying the cell, which can be difficult to do for less studied microorganisms. MPE is easier to implement than metabolic engineering and should have broad applications in biotechnology for the production of chemicals, fuels, and pharmaceuticals.

\section{References}

1. Silicon Valley Bank (2012) Silicon Valley Bank Cleantech Practice. The Advanced Biofuel and Biochemical Overview.

2. Fonseca RR, Schmitz JE, Fileti AM, da Silva FV (2013) A fuzzy-split range control system applied to a fermentation process. Bioresour Technol 142: 475 482

3. Mariano AP, Costa CB, Maciel MR, MaugeriFilho F, Atala DI, et al. (2010) Dynamics and control strategies for a butanol fermentation process. Appl Biochem Biotechnol 160:2424-2448.

4. Du C, Yan H, Zhang Y, Li Y, Cao Z (2006) Use of oxidoreduction potential as an indicator to regulate 1,3-propanediol fermentation by Klebsiella pneumoniae. Appl Microbiol Biotechnol 69: 554-563.

5. Peris M, Escuder-Gilabert L (2013) On-line monitoring of food fermentation processes using electronic noses and electronic tongues: a review. Anal ChimActa 804: 29-36.

6. Johnsson O, Andersson J, Liden G, Johnsson C, Hagglund T (2013) Feed rate control in fed-batch fermentations based on frequency content analysis. Biotechnol Prog 29: 817-824.

7. Heluane H, Evans MR, Dagher SF, Bruno-Barcena JM (2011) Meta-analysis and functional validation of nutritional requirements of solventogenic Clostridia growing under butanol stress conditions and coutilization of D-glucose and D-xylose. Appl Environ Microbiol 77: 4473-4485.

8. Wang Y, Li X, Blaschek HP (2013) Effects of supplementary butyrate on butanol production and the metabolic switch in Clostridium beijerinckii NCIMB 8052: genome-wide transcriptional analysis with RNA-Seq. Biotechnol Biofuels 6(1): 138.

9. Quek LE, Wittmann C, Nielsen LK, Kromer JO (2009) OpenFLUX: efficient modelling software for $13 \mathrm{C}$-based metabolic flux analysis. Microb Cell Fact $8: 25$. 
Citation: Yang ST, Liu X (2014) Metabolic Process Engineering for Biochemicals and Biofuels Production. J Microb Biochem Technol 6: e116. doi:10.4172/1948-5948.1000e116

Page 3 of 3

10. Zamboni N, Fischer E, Sauer U (2005) FiatFlux--a software for metabolic flux analysis from 13C-glucose experiments. BMC Bioinformatics 6: 209

11. Kohlstedt M, Becker J, Wittmann C (2010) Metabolic fluxes and beyond systems biology understanding and engineering of microbial metabolism. App Microbiol Biotechnol 88: 1065-1075.

12. Buchel F, Rodriguez N, Swainston N, Wrzodek C, Czauderna T, et al. (2013)
Path2Models: large-scale generation of computational models from biochemical pathway maps. BMC Syst Biol 7: 116

13. Hyduke DR, Lewis NE, Palsson BO (2013) Analysis of omics data with genomescale models of metabolism. Mol Biosyst 9: 167-174.

14. http://www.ebi.ac.uk/biomodels-main/path2models. 